\title{
Biomarkers for Identifying First-Episode Schizophrenia Patients Using Diffusion Weighted Imaging
}

\author{
Yogesh Rathi ${ }^{1,2,4}$, James Malcolm ${ }^{2}$, Oleg Michailovich ${ }^{3}$, Jill Goldstein ${ }^{1}$, \\ Larry Seidman $^{1}$, Robert W. McCarley ${ }^{4}$, Carl-Fredrik Westin ${ }^{1}$, \\ and Martha E. Shenton ${ }^{1,4}$ \\ 1 Harvard Medical School, Boston \\ ${ }^{2}$ Georgia Institute of Technology, Atlanta \\ 3 University of Waterloo, Canada \\ 4 VA Clinical Neuroscience Division, Brockton
}

\begin{abstract}
Recent advances in diffusion weighted MR imaging (dMRI) has made it a tool of choice for investigating white matter abnormalities of the brain and central nervous system. In this work, we design a system that detects abnormal features (biomarkers) of first-episode schizophrenia patients and then classifies them using these features. We use two different models of the dMRI data, namely, spherical harmonics and the two-tensor model. The algorithm works by first computing several diffusion measures from each model. An affine-invariant representation of each subject is then computed, thus avoiding the need for registration. This representation is used within a kernel based feature selection algorithm to determine the biomarkers that are statistically different between the two populations. Confirmation of how well these biomarkers identify each population is obtained by using several classifiers such as, k-nearest neighbors, Parzen window classifier, and support vector machines to separate 21 first-episode patients from 20 age-matched normal controls. Classification results using leave-manyout cross-validation scheme are given for each representation. This algorithm is a first step towards early detection of schizophrenia.
\end{abstract}

\section{Introduction}

A recent World Health Organization (WHO) report estimates that nearly $1 \%$ of the population in the US is affected by schizophrenia. A growing body of evidence suggests that early detection and treatment of schizophrenia (and many other brain disorders) is critical in forming and predicting the course and outcome of the disorder [1]. The tools proposed in this work can serve as a first step towards early detection of schizophrenia, which may result in better prognosis and functional outcome. However, very little work has been done on developing a biomarker that characterizes first-episode schizophrenia or other subtle psychiatric disorders such as mild to moderate traumatic brain injury.

There has been some work done on classifying patients with chronic schizophrenia using structural MRI [2|3]. The authors in [4|5] use dimensionality reduction followed by linear discriminant analysis for classification of patients with schizophrenia (chronic). They, however, only use the fractional anisotropy (FA) images derived from single tensor estimation as a discriminant feature. Another related work is by [6], where

T. Jiang et al. (Eds.): MICCAI 2010, Part I, LNCS 6361, pp. 657-665, 2010.

(c) Springer-Verlag Berlin Heidelberg 2010 
the authors use kernel methods for discriminating schizophrenia patients. Recent work has also focussed on using other imaging modalities, such as, functional MRI for detection of schizophrenia in prodromals [7]. The work presented in this paper, can provide complementary anatomical input to such fMRI based techniques for early detection of schizophrenia.

\section{Our Contribution}

In this work, we propose to design an algorithm that locates abnormal features of firstepisode (FE) schizophrenia patients. These features are then used within a classification system to determine their potential use as biomarkers. While several studies have reported statistical differences in diffusion measures for FE patients [89], to the best of our knowledge, this is the first study that uses them to perform classification.

Existing work on distinguishing chronic schizophrenia used the single tensor model, which is known to be inadequate in regions of crossing and branching - a common configuration occurring throughout the brain [10]. In this work, we use a nonparametric spherical harmonics model [11], as well as a parametric two-tensor model [12] to detect biomarkers and perform classification of FE patients. These models can better capture multi-fiber configurations and hence the abnormality in the underlying anatomy.

Another novel aspect in this work is the use of an affine invariant probabilistic representation of each subject, which avoids the computational cost of registration along with errors due to mis-registration. Finally, we use a kernel based method [13] to locate statistically different diffusion measures (biomarkers) followed by classification of FE patients using several classifiers, i.e., Parzen window classifier, k-nearest neighbor and support vector machines.

\section{Preliminaries}

Schizophrenia is characterized by several symptoms such as, hallucinations, delusions, suspiciousness, etc. These occur in varying degrees in people affected by this disorder. Chronic schizophrenia typically implies that the patient has had psychotic symptoms for atleast five years, while first-episode schizophrenics are patients who have recently had their first psychotic episode (thus they are in the early stage of the development of the disease). Thus, knowing anatomical biomarkers that can reliably distinguish FE patients can be quite useful in determining the risk for prodromal subjects (subjects with high risk of schizophrenia). Recent studies have shown that around $30-40 \%$ of prodromals convert into schizophrenics. Thus, a tool that can provide the probability of a prodromal subject being anatomically close to FE patients can be immensely useful for early detection of schizophrenia. This is the main motivation behind our work.

In diffusion weighted imaging, image contrast is related to the strength of water diffusion along fiber orientation. At each image voxel, diffusion is measured along a set of distinct gradients, $\mathbf{u}_{1}, \ldots, \mathbf{u}_{n} \in \mathbb{S}^{2}$ (on the unit sphere), producing the corresponding signal, $\mathbf{s}=\left[s_{1}, \ldots, s_{n}\right]^{T} \in \mathbb{R}^{n}$. One of the simplest model that explains $\mathbf{s}$ is the diffusion tensor model, which provides a Gaussian estimate of the fiber orientation [14]. However, this model is highly inadequate in regions of crossings and 
branching fibers [15]. To overcome this limitation, several other models have been pro-

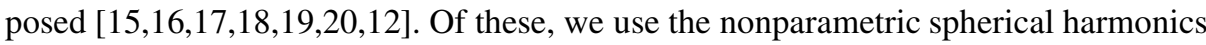
(SH) model of computing the orientation distribution funciton (ODF) [11] and the unscented Kalman filter (UKF) based two-tensor model proposed in [12]. These choices are guided by the following considerations: 1). The SH model can represent an arbitrary number of fiber configurations at each voxel, 2). The UKF based two-tensor model estimation incorporates the correlation in diffusion of water along the fiber direction, and thus is a robust estimator of the diffusion profile for one and two fiber configurations.

Spherical harmonics (SH): This nonparametric model is one of the popular techniques for estimating the diffusion ODF [11]. The method works by first computing the coefficients of the spherical harmonic ( $\mathrm{SH})$ basis of order $L$ that best fits the measured signal and subsequent analytical computation to obtain the ODF. Given any bandlimited signal $\mathbf{s}$ defined on the sphere, one can write it as an expansion in terms of the SH basis as: $s_{i}=\sum_{l=0}^{L} \sum_{m=-l}^{l} c_{l, m} Y_{l, m}$, where $Y_{l, m}$ are the spherical harmonic basis functions. The above equations can be written as a linear system of equations and $c_{l, m}$ can be computed using least squares.

Filtered Two-tensor (F2T): In this case, the signal is assumed to be generated by a mixture of two Gaussians. Thus, $s_{i}=\sum_{j=1}^{2} \exp \left(-b \mathbf{u}_{i}^{T} D_{j} \mathbf{u}_{i}\right)$, where $\left\{D_{1}, D_{2}\right\}$ are the two diffusion tensors estimated recursively within an unscented Kalman filtering framework [12]. The method works by starting tractography from the seed region and diffusion tensors are estimated as a fiber is traced from seed to termination. In this work, we perform whole brain tractography, by seeding the entire brain (except CSF). Thus, the F2T model is estimated at every voxel of the brain (in terms of the fibers that pass through each voxel), apart from CSF.

\section{Methods}

Validation studies have indicated the correlation between de-myelination, cellular packing, and axonal damage to diffusion measures such as fractional anisotropy (FA), trace (TR), norm (N), etc., [21|22]. Thus, these measures are potential candidates for being used as biomarkers. In the case of the SH model, generalized fractional anisotropy (GFA) and generalized norm (GN) are the diffusion measures of interest [23]. These measures can be readily computed in the $\mathrm{SH}$ basis as follows 1 :

$$
\mathrm{GFA}=\sqrt{\frac{n \sum_{i=1}^{n}\left(S_{i}-\bar{S}\right)^{2}}{(n-1) \sum_{i=1}^{n} S_{i}^{2}}}, \quad \mathrm{GN}=\|\mathbf{c}\|_{2}, \text { with } \mathbf{S}=\left[S_{1} S_{2} \ldots S_{n}\right],
$$

where $\mathbf{S}$ is the estimated signal using the $\mathrm{SH}$ model, $\mathbf{c}=\left[c_{1} c_{2} \ldots\right]$ are the coefficients in the SH basis, and $n$ is the number of samples. GFA captures the anisotropy of the signal, while GN measures the "size" or amount of diffusion. Note that, these measures are typically used for ODF's, but given the linear relation between the signal and the $\mathrm{ODF}$ in the $\mathrm{SH}$ basis [11], the measures computed above are equivalent (upto a linear transformation) to those computed for ODF's. Computing these measures directly for the signal avoids computation of the ODF.

\footnotetext{
${ }^{1}$ The acquired scanner signal is denoted by $\mathbf{s}$, while the estimated signal is denoted by $\mathbf{S}$.
} 
The F2T model allows for computation of a different set of orthogonal diffusion measures such as the FA, Mode (MD) and norm (N) [24]. These measures capture different (orthogonal) aspects of the shape of the tensor. Thus, FA measures the anisotropy while norm captures the amount of diffusion. Mode distinguishes between planar, ellipsoidal and spherical shapes. Given, a diffusion tensor $D$, these measures can be computed as follows: $\mathrm{N}=\|D\|, \quad \mathrm{FA}=\frac{\sqrt{3} \tilde{D}}{\sqrt{2}\|D\|}, \quad \mathrm{MD}=3 \sqrt{6}\left|\left(\frac{\tilde{D}}{\|\tilde{D}\|}\right)\right|, \quad \tilde{D}=D-\frac{1}{3} \operatorname{tr}(D) I$

where, $|$.$| denotes the determinant, \operatorname{tr}($.$) is the trace, I$ is the identity matrix and $\|$. $\|$ denotes the frobenius norm of a matrix. These measures can be computed for each tensor, and thus six features are obtained.

\subsection{Affine Invariant Representation}

The next step in our algorithm is computing an affine invariant representation, i.e., a representation that does not change even if an affine transformation is applied to the underlying data. We achieve this by computing a probability density function (PDF) of each diffusion measure defined above. A nonparametric estimate of the PDF can be computed using the following expression [25]: $p(z)=\frac{1}{M h} \sum_{i=1}^{M} G\left(\frac{z-I(x)}{h}\right), \quad z \in$ Range of $I\}$, where $I(x)$ is a scalar value at spatial location $x, M$ is the number of data points, $G$ is a Gaussian kernel and $h$ denotes the bandwidth of the kernel. Notice that, the spatial position $x$ is arbitrary and applying an affine transformation to it does not change the PDF.

For each of the diffusion measures discussed earlier, we compute a PDF from values estimated throughout the brain. Thus, for the $\mathrm{SH}$ model, an affine invariant representation of a subject is given by: $\mathbf{P}_{s h}=\left[p_{g f a} p_{g n}\right]$, where $p_{g f a}$ and $p_{g n}$ are the PDF's of the GFA and GN respectively. The PDF's are computed at $n_{b}$ bins, and thus $\mathbf{P}_{s h}$ is an $n_{b} \times 2$ matrix. Similarly, for the F2T model, we compute PDF's for all the 6 diffusion measures to obtain a probabilistic representation $\mathbf{P}_{f 2 t}=\left[\begin{array}{lllll}p_{f a_{1}} & p_{m d_{1}} & p_{n_{1}} & p_{f a_{2}} & p_{m d_{2}} \\ p_{n_{2}}\end{array}\right]$ of each subject $\left(n_{b} \times 6\right.$ matrix).

\subsection{Biomarker Detection and Classification}

Once a PDF of each diffusion measure is computed for each subject, the goal is to determine, which of these measures characterize FE patients. Since each PDF is a high $\left(n_{b}\right)$ dimensional vector, appropriate methods have to be used. As such, we will use the kernel based method of [13] for statistical hypothesis testing. This method has several advantages, chief among them are : a) It can be used with high dimensional data without sacrificing robustness and accuracy. b) The data need not necessarily lie in a Euclidean vector space, i.e., any type of data with an appropriate kernel can be used. c) This method computes statistical differences without any assumption on the distribution from which the samples are drawn (the popular t-test assumes a Gaussian distribution for the samples in each population). Thus, subtle differences can be captured using the kernel based method.

This method tests the hypothesis of two distributions being equal $p=q$. The test statistic used is the maximum mean discrepancy (MMD) between the two samples. Let 
$\mathcal{F}$ be a class of functions $f: \mathcal{X} \rightarrow \mathbb{R}$ and $p, q$ be probability distributions (with domain $\mathcal{X})$, then MMD is defined as: $M M D[\mathcal{F}, p, q]=\sup _{f \in \mathcal{F}}\left(\mathbb{E}_{x \sim p}[f(x)]-\mathbb{E}_{y \sim q}[f(y)]\right)$, where $\mathbb{E}$ represents the expected value. Computing MMD involves, mapping the data to a reproducing kernel Hilbert space (RKHS) and computing the inner product (between high dimensional features) in this space using an appropriate kernel (Gaussian in our case). If MMD is greater than a certain threshold, the null hypothesis $(p=q)$ is rejected. The hypothesis threshold is selected based on significance level $\alpha$, typically set to 0.05 .

We will use this kernel method for feature selection (biomarker detection). Thus, in the case of F2T model, each of the PDF's $p_{i} \in \mathbf{P}_{f 2 t}$ is tested, and the ones which are statistically different are used for classification. Classification involves learning a function that minimizes a particular metric so as to best separate the groups in the training data set. Several classifiers have been proposed in the literature. We will use three popular ones: Parzen window classifier [26], k-nearest neighbor [27] and support vector machines (SVM) [28]. A typical way to ensure robustness to overfitting for any classifier is to perform a leave-many-out cross validation. In this technique, a certain percentage of the available data are randomly selected (without replacement) for training the classifier. Testing is then performed on the remaining data and classification error computed. This process is repeated several thousand times and the overall performance of the classifier is computed in terms of its sensitivity and specificity.

Briefly, the entire algorithm can be summarized as follows:

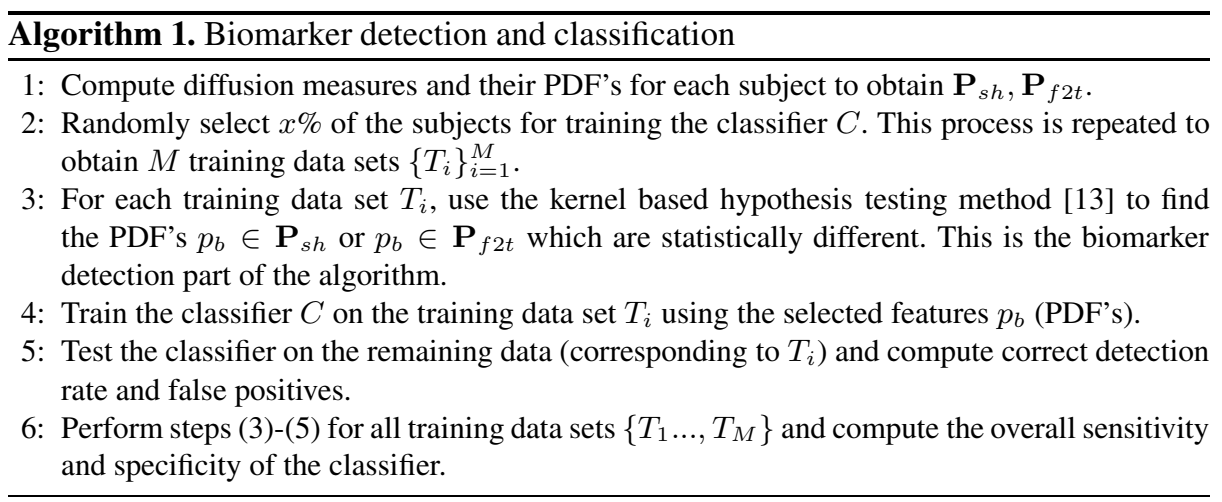

In step 3 above, a counter (corresponding to each diffusion measure) is incremented each time a diffusion measure is selected as statistically different. Thus the measure that best characterizes a given population will be chosen frequently, thereby indicating its potential use as a biomarker.

\section{Results}

We applied the above algorithm for detecting biomarkers of FE schizophrenia patients. The data set consisted of $21 \mathrm{FE}$ patients (17 males, 4 females, average age: $21.21 \pm 4.56$ years) and 20 normal controls ( 15 males, 5 females, average age: $22.47 \pm 3.48$ years) 
Table 1. Classification accuracy for SH and F2T models

\begin{tabular}{|c|c|c|c|c|c|c|c|c|c|c|c|c|c|c|c|c|}
\hline \multirow{3}{*}{ Classifier } & \multicolumn{8}{|c|}{ SH model } & \multicolumn{8}{|c|}{ F2T model } \\
\hline & \multicolumn{2}{|c|}{$40 \%$} & \multicolumn{2}{|c|}{$60 \%$} & \multicolumn{2}{|c|}{$80 \%$} & \multicolumn{2}{|c|}{$98 \%$} & \multicolumn{2}{|c|}{$40 \%$} & \multicolumn{2}{|c|}{$60 \%$} & \multicolumn{2}{|c|}{$80 \%$} & \multicolumn{2}{|c|}{$98 \%$} \\
\hline & $\mathrm{Sp}$ & $\mathrm{Se}$ & $\mathrm{Sp}$ & $\mathrm{Se}$ & $\mathrm{Sp}$ & $\mathrm{Se}$ & $\mathrm{Sp}$ & $\mathrm{Se}$ & $\mathrm{Sp}$ & $\mathrm{Se}$ & $\mathrm{Sp}$ & $\mathrm{Se}$ & Sp & $\mathrm{Se}$ & Sp & $\mathrm{Se}$ \\
\hline knn & 0.71 & 0.72 & 0.76 & 0.76 & 0.78 & 0.77 & 0.80 & 0.78 & 0.83 & 0.82 & 0.85 & 0.85 & 0.85 & 0.85 & 0.85 & 0.86 \\
\hline SVM & 0.70 & 0.69 & 0.75 & 0.71 & 0.77 & 0.73 & 0.77 & 0.77 & 0.79 & 0.78 & 0.83 & 0.80 & 0.84 & 0.81 & 0.85 & 0.81 \\
\hline PWC & 0.70 & 0.72 & 0.70 & 0.76 & 0.68 & 0.79 & 0.70 & 0.84 & 0.79 & 0.77 & 0.80 & 0.85 & 0.82 & 0.87 & 0.80 & 0.85 \\
\hline
\end{tabular}

with the p-value for age being 0.34. dMRI data was acquired for all the subjects on a $3 \mathrm{~T}$ scanner with 51 gradient encoding directions and 8 baseline images. The spatial resolution was $1.66 \times 1.66 \times 1.7 \mathrm{~mm}^{3}$ with a b-value of 900 .

The SH model was estimated throughout the brain, while F2T model estimation was done in terms of whole brain tractography [12]. Thus, F2T was not estimated in CSF areas, where no fiber tracts exist. Estimation of both these models was done for all 41 subjects and the proposed algorithm was applied as described before (Algorithm 1).

For the SH model, the generalized norm (GN) was consistently chosen as the biomarker by the kernel hypothesis testing method [13] for all the training data sets. The reason is evident from the plots of PDF's of all subjects (see Figure 1). In the case of F2T model, norm of the two tensors (N1, N2) were the distinguishing features that separated the two groups. No differences in FA or mode were observed. Thus, for both the models, a measure of the "amount of diffusion" was chosen as the biomarker that characterized FE patients, i.e., overall diffusivity in FE patients is higher than NC. Biologically, this implies that, in the FE patients there is demyelination of the axons or lower density of

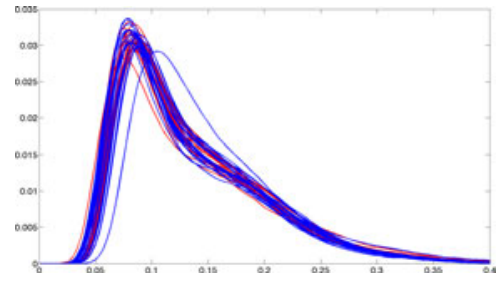

GFA

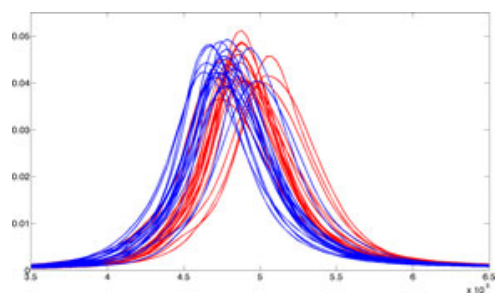

N1

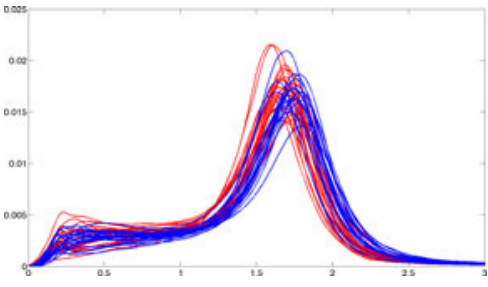

GN

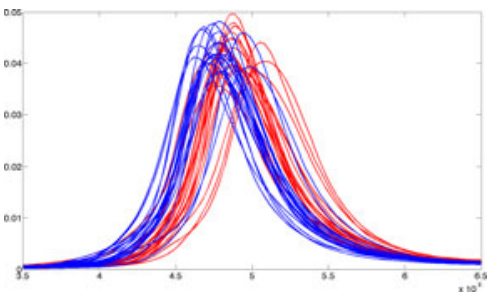

$\mathrm{N} 2$

Fig. 1. PDF's of diffusion measures for $21 \mathrm{FE}$ patients (red) and $20 \mathrm{NC}$ (blue). Top row: GFA and GN are shown, but only GN was chosen as biomarker for the SH model. Bottom: Norms of both the tensors in F2T model show significant difference between the groups. 
cellular packing, allowing for more diffusion of water. Another point to note, as evident in Figure 1, is that the tensor norms $(\mathrm{N} 1, \mathrm{~N} 2)$ measure the diffusivity, which is higher for FE patients, while the signal norm $(\mathrm{GN})$ is lower for FE patients. This is because, higher diffusivity $D$ implies lower signal $S: S\left(\mathbf{u}_{i}\right)=S_{0} \exp \left(-b D\left(\mathbf{u}_{i}\right)\right)$.

Table 1 gives the sensitivity (Se) and specificity $(\mathrm{Sp})$ of each of the classifier's (for both models) with different number of samples $(x=\{40 \%, 60 \%, 80 \%, 98 \%\})$ in the training data set. For each $x, 10000$ training data sets were randomly generated from the original data and testing was done on the remaining samples. This method of crossvalidation is a very good estimator of the generalization property of any classifier [29].

For the k-nearest neighbor (knn) classifier, we used 6 nearest neighbors, with cosine of the angle between the PDF's as a measure of similarity (the PDF's can be thought of as $n_{b}$ dimensional vectors). In the case of SVM and Parzen window classifier (PWC), the kernel width was chosen so as to minimize the error during training.

In general, the k-nearest neighbor $(\mathrm{knn})$ classifier gave the best performance for this data set. Also, the F2T model performed much better in terms of the classification accuracy than the SH model. Notice that, the performance of knn is close to optimal even when only $60 \%$ of the data is used in the training data set. Combining the features from both the models did not improve the performance of any classifier.

\section{Discussion and Application}

In this work, we proposed a system for detecting biomarkers of FE schizophrenia patients using two representations; spherical harmonics and two-tensor. The effectiveness of using these biomarkers to characterize FE patients was obtained by testing their classification accuracy ( $85 \%$ specificity and $86 \%$ sensitivity). Future application involves using these biomarkers to determine the probability of a prodromal subject being at risk of developing schizophrenia. This can be easily done by computing $\hat{P}=\left[p_{n_{1}}, p_{n_{2}}\right]$ for a prodromal subject and then using a nonparametric density estimator to compute the probability of $\hat{P}$ being a FE patient. This will be the focus of our future work. Thus, the proposed method can be of great clinical significance for early detection of schizophrenia.

\section{Acknowledgements}

This work was supported in part by a Department of Veteran Affairs Merit Award (Dr. M Shenton, Dr. R McCarley), the VA Schizophrenia Center Grant (RM, MS) and NIH grants:1P50MH080272-01 (MS,RM), P41 RR13218 (MS), R01 MH 52807 (RM), R01 MH 50740 (MS), R01MH074794 (Dr. Westin) and NA-MIC (NIH) grant U54 GM072977-01 (Dr. Ron Kikinis).

\section{References}

1. McGlashan, T.: Early detection and intervention in schizophrenia: editors introduction. Schizophr Bull 22(2), 197-199 (1996)

2. Davatzikos, C., Shen, D., Gur, R., Wu, X., Liu, D., Fan, Y., Hughett, P., Turetsky, B., Gur, R.: Whole-brain morphometric study of schizophrenia revealing a spatially complex set of focal abnormalities. Archives of general psychiatry 62(11), 1218-1227 (2005) 
3. Pohl, K.M., Sabuncu, M.R.: A unified framework for $\mathrm{mr}$ based disease classification. In: Prince, J.L., Pham, D.L., Myers, K.J. (eds.) IPMI 2009. LNCS, vol. 5636, pp. 300-313. Springer, Heidelberg (2009)

4. Caan, M., Vermeer, K., van Vliet, L., Majoie, C., Peters, B., den Heeten, G., Vos, F.: Shaving diffusion tensor images in discriminant analysis: A study into schizophrenia. Medical Image Analysis 10(6), 841-849 (2006)

5. Caprihan, A., Pearlson, G., Calhoun, V.: Application of principal component analysis to distinguish patients with schizophrenia from healthy controls based on fractional anisotropy measurements. Neuroimage 42(2), 675-682 (2008)

6. Khurd, P., Verma, R., Davatzikos, C.: Kernel-based manifold learning for statistical analysis of diffusion tensor images. In: Karssemeijer, N., Lelieveldt, B. (eds.) IPMI 2007. LNCS, vol. 4584, p. 581. Springer, Heidelberg (2007)

7. Schobel, S., Lewandowski, N., Corcoran, C., Moore, H., Brown, T., Malaspina, D., Small, S.: Differential targeting of the CA1 subfield of the hippocampal formation by schizophrenia and related psychotic disorders. Archives of General Psychiatry 66(9), 938 (2009)

8. Friedman, J., Tang, C., Carpenter, D., Buchsbaum, M., Schmeidler, J., Flanagan, L., Golembo, S., Kanellopoulou, I., Ng, J., Hof, P., et al.: Diffusion tensor imaging findings in first-episode and chronic schizophrenia patients. American Journal of Psychiatry 165(8), 1024 (2008)

9. Kubicki, M., McCarley, R., Westin, C.F., Park, H.J., Maier, S., Kikinis, R., Jolesz, F., Shenton, M.: A review of diffusion tensor imaging studies in schizophrenia. J. of Psychiatric Research 41, 15-30 (2007)

10. Behrens, T., Johansen-Berg, H., Jbabdi, S., Rushworth, M., Woolrich, M.: Probabilistic diffusion tractography with multiple fibre orientations: What can we gain? NeuroImage 34, 144-155 (2007)

11. Descoteaux, M., Angelino, E., Fitzgibbons, S., Deriche, R.: Regularized, fast, and robust analytical Q-ball imaging. Magnetic Resonance in Medicine 58, 497-510 (2007)

12. Malcolm, J., Shenton, M., Rathi, Y.: Neural tractography using an unscented Kalman filter. In: Prince, J.L., Pham, D.L., Myers, K.J. (eds.) Information Processing in Medical Imaging. LNCS, vol. 5636, pp. 126-138. Springer, Heidelberg (2009)

13. Gretton, A., Borgwardt, K., Rasch, M., Scholkopf, B., Smola, A.: A kernel method for the two-sample-problem. Journal of Machine Learning Research 1, 1-10 (2008)

14. Basser, P., Mattiello, J., LeBihan, D.: MR diffusion tensor spectroscopy and imaging. Biophysical Journal 66(1), 259-267 (1994)

15. Tuch, D.: Q-ball imaging. Magnetic Resonance in Medicine 52, 1358-1372 (2004)

16. Anderson, A.: Measurement of fiber orientation distributions using high angular resolution diffusion imaging. Magnetic Resonance in Medicine 54(5), 1194-1206 (2005)

17. Jian, B., Vemuri, B.: A unified computational framework for deconvolution to reconstruct multiple fibers from diffusion weighted MRI. TMI 26(11), 1464-1471 (2007)

18. Jansons, K., Alexander, D.: Persistent angular structure: New insights from diffusion MRI data. Inverse Problems 19, 1031-1046 (2003)

19. Tournier, J.D., Calamante, F., Gadian, D., Connelly, A.: Direct estimation of the fiber orientation density function from diffusion-weighted MRI data using spherical deconvolution. NeuroImage 23, 1176-1185 (2004)

20. Barmpoutis, A., Hwang, M.S., Howland, D., Forder, J., Vemuri, B.: Regularized positivedefinite fourth order tensor field estimation from DW-MRI. Neuroimage (2009)

21. Budde, M., Kim, J., Liang, H., Schmidt, R., Russell, J., Cross, A., Song, S.: Toward accurate diagnosis of white matter pathology using diffusion tensor imaging. Magnetic resonance in medicine 57(4), 688 (2007)

22. Chenevert, T., Brunberg, J., Pipe, J.: Anisotropic diffusion in human white matter: demonstration with MR techniques in vivo. Radiology 177(2), 401-405 (1990) 
23. Ozarslan, E., Vemuri, B., Mareci, T.: Generalized scalar measures for diffusion MRI using trace, variance, and entropy. Magnetic Resonance in Medicine 53(4), 866-876 (2005)

24. Kindlmann, G., Ennis, D., Whitaker, R., Westin, C.: Diffusion tensor analysis with invariant gradients and rotation tangents. TMI 26(11), 1483-1499 (2007)

25. Parzen, E.: On estimation of a probability density function and mode. The annals of mathematical statistics, 1065-1076 (1962)

26. Jain, A., Ramaswami, M.: Classifier design with Parzen windows. Pattern Recognition and artificial intelligence, 211-228 (1988)

27. Cover, T., Hart, P.: Nearest neighbor pattern classification. IEEE Transactions on Information Theory 13(1), 21-27 (1967)

28. Schölkopf, B., Burges, C., Smola, A.: Advances in kernel methods: support vector learning. MIT Press, Cambridge (1999)

29. Picard, R., Cook, R.: Cross-validation of regression models. Journal of the American Statistical Association 79(387), 575-583 (1984) 